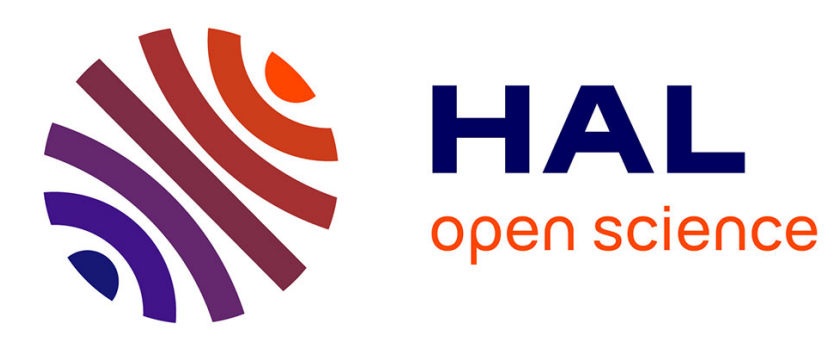

\title{
Mesure par pénétrométrie de viscosités élevées (104-1011 Pl) à basse température (77-300 K)
}

Alberte Bondeau, J. Huck

\section{To cite this version:}

Alberte Bondeau, J. Huck. Mesure par pénétrométrie de viscosités élevées (104-1011 Pl) à basse température $(77-300 \mathrm{~K})$. Revue de Physique Appliquée, 1983, 18 (5), pp.307-312. 10.1051/rphysap:01983001805030700 . jpa-00245096

\section{HAL Id: jpa-00245096 https://hal.science/jpa-00245096}

Submitted on 1 Jan 1983

HAL is a multi-disciplinary open access archive for the deposit and dissemination of scientific research documents, whether they are published or not. The documents may come from teaching and research institutions in France or abroad, or from public or private research centers.
L'archive ouverte pluridisciplinaire HAL, est destinée au dépôt et à la diffusion de documents scientifiques de niveau recherche, publiés ou non, émanant des établissements d'enseignement et de recherche français ou étrangers, des laboratoires publics ou privés. 


\title{
Mesure par pénétrométrie de viscosités élevées $\left(10^{4}-10^{11} \mathrm{PI}\right)$ à basse température (77-300 K)
}

\author{
A. Bondeau \\ Laboratoire des diélectriques liquides, U.E.R. de Physique, \\ Université Lyon I, 43, bd du 11-Novembre-1918, 69622 Villeurbanne Cedex, France
}

et J. Huck

Laboratoire de Physique des Matériaux, U.E.R. de Sciences, Université de Saint-Etienne, 23, rue du Docteur-Paul-Michelon, 42023 Saint-Etienne Cedex, France

(Reçu le 27 décembre 1982, accepté le 17 février 1983)

\begin{abstract}
Résumé. - On a réalisé un pénétromètre à cylindres destiné à la mesure des viscosités de cisaillement des liquides newtoniens comprises entre $10^{4}$ et $10^{11} \mathrm{Pl}$ dans l'intervalle de température $77-300 \mathrm{~K}$. L'évolution temporelle de la profondeur d'enfoncement $h(t)$ mesurée par un capteur électromagnétique de déplacement est enregistrée sur mémoire magnétique et traitée par un calculateur de table. Les variations de $h^{2}(t)$ sont linéaires conformément à la théorie. Les valeurs de la viscosité du phényle salicylate mesurées avec ce dispositif sont en bon accord avec celles de Laughlin et Uhlmann obtenues par flexion d'une éprouvette.
\end{abstract}

\begin{abstract}
We have achieved a cylindric penetrometer in order to measure Newtonian liquids shear viscosity between $10^{4}-10^{11} \mathrm{~Pa}$.s and 77-300 K. Measurements of the penetration depth $h(t)$ by means of an electromagnetic displacement captor are recorded on a magnetic memory and treated by a desktop computer. The variations of $h^{2}(t)$ are linear according to the theory. The viscosity values of phenyl salicylate measured by this penetrometer agree with the data of Laughlin and Uhlmann obtained by beam bending method.
\end{abstract}

1. Introduction. - Il est bien connu que l'extrapolation des différentes lois empiriques d'ajustement des variations de la viscosité des liquides en fonction de la température est souvent hasardeuse et peut conduire à des erreurs considérables [1]. D'autre part les modèles mathématiques de la viscosité s'appliquent relativement mal aux liquides associés ou aux réseaux à liaison covalente [2] et pour cette raison il est toujours préférable de mesurer la viscosité dans une large plage de température et d'éviter ainsi toute extrapolation.

Dans le domaine des liquides surfondus à basse température, les mesures des viscosités de cisaillement élevées, de l'ordre de $10^{7}$ à $10^{12} \mathrm{Pl}$ sont peu nombreuses [3-8], et cela malgré l'intérêt qu'elles peuvent présenter pour tester les différents modèles théoriques de la transition vitreuse [9]. Les techniques qui ont été utilisées dans cette gamme de viscosité par différents auteurs sont basées sur la déformation d'une éprouvette soumise à une contrainte [7], l'extrusion $[4,5]$ ou la viscosimétrie à rotation $[8,10]$. Dans ce dernier cas une amélioration certaine a été apportée récem- ment par Berberian [10] qui a utilisé un système capacitif pour mesurer les petits angles de rotation.

Pour la mesure des hautes viscosités on doit tenir compte de la durée d'observation qui est nécessairement élevée, puisque dans la zone proche de la température de transition vitreuse $T_{\mathrm{g}}$ (définie par la valeur $10^{12} \mathrm{Pl}$ de la viscosité $\eta$ ), le module de cisaillement $G$ des liquides surfondus vaut environ $10^{9} \mathrm{~Pa}$ [1] et que le temps de relaxation $\tau_{M}=\eta G^{-1}$ est de l'ordre de $10^{3} \mathrm{~s}$. Il faut ainsi prévoir des durées d'observation grandes devant $\tau_{\mathrm{M}}$, et il paraît donc indispensable de procéder à l'automatisation des mesures et à leur traitement informatisé. C'est la méthode que nous avons utilisée en choisissant pour des raisons de simplicité mécanique la technique de pénétrométrie. En général les pénétromètres commercialisés fonctionnent à température ambiante avec différents corps d'épreuves, cônes, sphères, cylindres, et c'est le temps de chute du corps à travers une épaisseur connue de liquide qui est mesuré $[11,12]$. Dans le cas d'une aiguille cylindrique, il est possible de procéder à des mesures absolues de la viscosité si l'on connaît les 
variations de la profondeur d'enfoncement $h(t) \mathrm{du}$ cylindre au cours du temps [13].

Le viscosimètre que nous avons réalisé comprend d'une part un cryostat à azote contenant le liquide et le système de pénétration, d'autre part un ensemble d'acquisition et de traitement de données constitué par une calculatrice de table pilotant un voltmètre digital couplé à un scanner multicanaux et une table traçante. L'enfoncement $h$ et la température du liquide sont mesurés à intervalle de temps réglable à l'aide de capteurs appropriés. Les informations prélevées par le voltmètre sont transmises au calculateur, stockées dans une mémoire, puis traitées. Ce dispositif nous a permis de mesurer des viscosités comprises entre $10^{3}$ et $10^{11} \mathrm{Pl}$ environ à l'aide d'enregistrements pouvant s'étaler selon les cas entre $10 \mathrm{~s}$ et plusieurs dizaines d'heures.

2. Bases théoriques de la pénétrométrie. - Le problème de l'enfoncement d'un cylindre de rayon $R_{1}$, soumis à une force constante, dans un liquide newtonien contenu dans un cylindre coaxial de rayon $\boldsymbol{R}_{\mathbf{2}}$ a été étudiée par différents auteurs [13-15]. Les résultats obtenus ont été utilisés par Smith et al. [13] pour caractériser les propriétés viscoélastiques en basse fréquence de solutions de polymères de faible viscosité $\left(\lesssim 10^{2} \mathrm{Pl}\right)$.

Sous l'effet de la pression exercée par le cylindre intérieur, le liquide s'écoule dans l'espace annulaire délimité par les deux cylindres coaxiaux. Dans le cas d'un liquide incompressible et d'un écoulement laminaire, l'équation de Navier Stokes [16, 17] en régime permanent conduit, en négligeant la pression engendrée par la hauteur du liquide à :

$$
\frac{\mathrm{d} p}{\mathrm{~d} z}=\eta \Delta v
$$

où $p$ est la pression exercée par le cylindre à la profondeur $h, v$ la vitesse du liquide, $\eta$ sa viscosité de cisaillement.

L'intégration de (1) en coordonnées cylindriques conduit à :

$$
v(r)=-\frac{p}{4 \eta h} r^{2}+A \ln r+B
$$

La pression $p$ et les constantes d'intégration $A$ et $B$ peuvent s'exprimer en fonction de la vitesse $\left(-v_{1}\right)$ du cylindre intérieur en utilisant les conditions aux limites :

$$
v\left(R_{1}\right)=-v_{1} \quad \text { et } \quad v\left(R_{2}\right)=0
$$

et l'équation de conservation du volume du liquide :

$$
v_{1} R_{1}^{2}=2 \int_{R_{1}}^{R_{2}} v r \mathrm{~d} r .
$$

Ce faisant on obtient la distribution radiale de la vitesse (Fig. 1) sous la forme suivante, où $x=R_{2} / R_{1}$ :

$$
\frac{v(r)}{v_{1}}=\frac{x^{2}\left[1-\left(r / R_{2}\right)^{2}\right]+\left(1+x^{2}\right) \ln \left(r / R_{2}\right)}{\left(1+x^{2}\right) \ln x+1-x^{2}}
$$

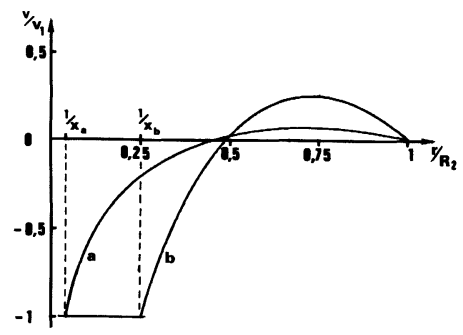

Fig. 1. - Distribution radiale de la vitesse du liquide au cours d'une expérience de pénétrométrie avec cylindres coaxiaux. a) $x=R_{2} / R_{1}=24$; b) $x=4$.

[Velocity radial distribution of the liquid during an experiment with coaxial cylinders. a) $x=R_{2} / R_{1}=24 ; b$ ) $x=4$.]

La viscosité est calculée en tenant compte de l'équilibre entre les forces de viscosité $2 \pi R_{1} h \eta(\partial v / \partial r)_{R_{1}}$, les forces de pression $\pi R_{1}^{2} p$ et le poids $F$ du cylindre. On obtient :

$$
\eta=\frac{1}{K} \frac{F}{v_{1} h}
$$

où $K$ est un facteur purement géométrique ne dépendant que du rapport des rayons des cylindres fixe et mobile (Fig. 2) :

$$
K=\frac{2 \pi\left(1+x^{2}\right)}{\left(1+x^{2}\right) \ln x+1-x^{2}} .
$$

Il résulte de (5) que la contrainte de cisaillement $\sigma$ est inversement proportionnelle à $h$ :

$$
\sigma=\frac{1}{2 \pi R_{1} h} \frac{x^{2}-1}{x^{2}+1} F .
$$

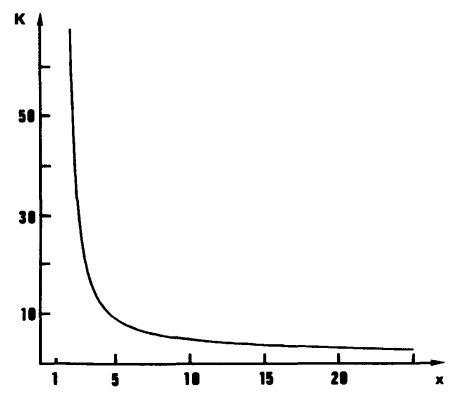

Fig. 2. - Variations, en fonction de $x=R_{2} / R_{1}$, du facteur géométrique $K$ du pénétromètre à cylindres coaxiaux.

[Variation of $K$ geometric factor with $x=R_{2} / R_{1}$ in a coaxial cylinders penetrometer.] 
Les variations de $h^{2}(t)$ sont linéaires puisque $v_{1}=\mathrm{d} h / \mathrm{d} t$. En posant $b=\mathrm{d} h^{2} / \mathrm{d} t$ et $C=2 F / K$, on obtient :

$$
\eta=\frac{C}{b}
$$

et la détermination de la viscosité se ramène ainsi à celle de la pente $b$ de $h^{2}(t)$.

3. Dispositif expérimental. - Le schéma de l'installation est représenté figure 3 . On distingue la cellule de mesure et l'équipage mobile, le cryostat et sa réserve d'azote liquide à niveau constant, le programmateur de température, le capteur de déplacement, le système d'acquisition et de traitement des données.

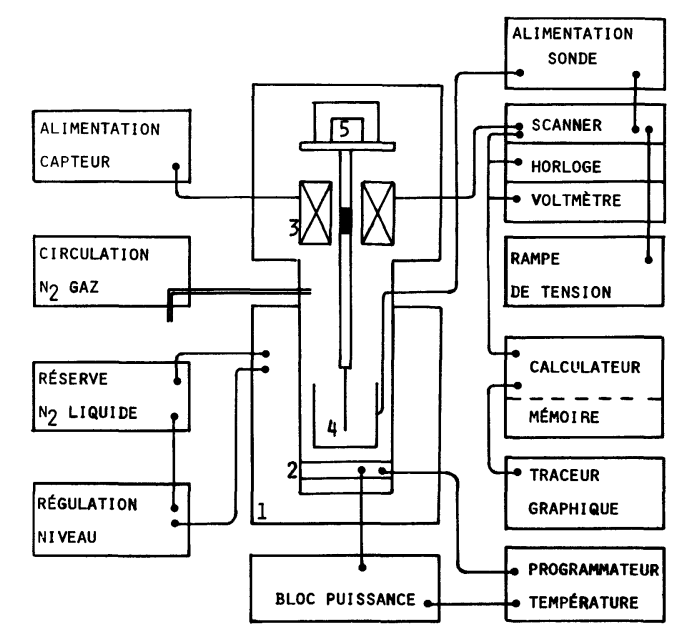

Fig. 3. - Schéma de principe du dispositif de mesure de viscosité par pénétrométrie. 1 : cryostat; 2 : thermaliseur; 3 : capteur de déplacement ; 4 : cellule ; $5:$ masse de charge.

[General arrangement of the penetroviscometer : 1 : cryostat $; 2:$ heating apparatus $; 3$ : displacement captor $; 4$ : cell ; 5 : load mass.]

3.1 Cellule De mesure. - Elle est constituée d'une nacelle cylindrique (diamètre intérieur $2 R_{2}=24 \mathrm{~mm}$, hauteur $40 \mathrm{~mm}$ ) contenant le liquide. Le cylindre intérieur est fixé à un équipage mobile d'axe vertical comprenant une tige en céleron, matériau choisi pour sa bonne isolation thermique, et le noyau ferromagnétique d'un capteur de déplacement. Un plateau horizontal peut être associé à cet ensemble et recevoir différentes masses. L'équipage mobile le plus léger a une masse de $37 \mathrm{~g}$, le plus lourd peut atteindre (avec le plateau chargé) une masse cent fois plus grande. Les cylindres de pénétration ont des diamètres compris entre $1 \mathrm{~mm}$ et $6 \mathrm{~mm}$, et une hauteur de $30 \mathrm{~mm}$. Le facteur géométrique correspondant est ainsi compris entre 4 et 24 (Fig. 2).

3.2 CRYostat. - Il s'agit d'un cryostat à convection construit par la Société des Matériels Cryogéniques, qui peut fonctionner entre 77 et $300 \mathrm{~K}$. La tem- pérature de l'azote gazeux est réglée par un thermaliseur, et la régulation thermique de l'ensemble est assurée par un programmateur de température ARION de type proportionnel-intégral-différentiel. Pour la mesure des températures on utilise des sondeš à résistance de platine CLARAL (norme DIN 43760) alimentées par un générateur de courant d'intensité constante $(1 \mathrm{~mA})$. La dérive de la température n'a jamais excédé $0,3 \mathrm{~K}$ au cours des expériences isothermes d'une durée supérieure à $24 \mathrm{~h}$.

3.3 CAPTEURS DE DÉPLACEMENT. - Nous utilisons deux capteurs de déplacement SCHAEVITZ de type HR.DC, dont la sensibilité est $2,68 \mathrm{~V} / \mathrm{mm}$ sur une distance de $9 \mathrm{~mm}$ pour l'un, et de $0,4 \mathrm{~V} / \mathrm{mm}$ sur $30 \mathrm{~mm}$ pour l'autre. Il s'agit de transformateurs différentiels à variations linéaires [18] constitués d'un noyau ferromagnétique cylindrique solidaire du système de déplacement, qui canalise le flux magnétique entre des bobinages fixes. Le signal induit par le capteur est proportionnel au déplacement du noyau. La résolution $(10 \mu \mathrm{V})$ du voltmètre digital (DATRON 1041) permet de repérer, avec le capteur le plus sensible, un déplacement de $3,7 \mu$.m.

3.4 ACQUISITION DES DONNÉES. - Le calculateur (HP 9815) est programmé pour suivre en fonction du temps la profondeur $h(t)$ et la température $T$ relevées par le voltmètre associé à un scanner (DATRON 1200). L'exécution du programme est lancée en coïncidence avec la libération de l'équipage mobile à $h=0$. Le déroulement de l'expérience est alors automatique et peut être ainsi schématisé :

- Relevé du temps $t$, de la tension $V$ à la sortie du capteur et de la tension $V_{\mathrm{T}}$ aux bornes de la sonde. Les délais séparant ces trois opérations sont très courts, de l'ordre de quelques millisecondes. En particulier les prélèvements de $t$ et $V$ se font sans changement de voie sur le scanner et peuvent, à l'échelle des manipulations les plus rapides, être considérés comme simultanés.

- Conversion de $V$ en $h$ et de $V_{\mathrm{T}}$ en $T$. $T(t)$.

- Report des points correspondants $h(t), h^{2}(t)$,

- Stockage sur mémoire secondaire (cassette) des quantités $h, t, T$, et impression.

- Bouclage sur un nouveau relevé, après une période d'échantillonnage $\Delta t$ réglable depuis $\Delta t=5 \mathrm{~s}$ pour les expériences les plus rapides jusqu'à $\Delta t$ de l'ordre de $30 \mathrm{~min}$. pour les plus lentes.

La base de temps utilisée dans le déroulement cidessus est interne au scanner. Sa résolution de $1 \mathrm{~s}$ exige une durée minimale de l'enfoncement voisine de $1 \mathrm{~min}$. ce qui correspond à une viscosité supérieure à $10^{4} \mathrm{Pl}$. Pour mesurer des viscosités plus faibles, on peut utiliser une base de temps donnée par la durée des cycles d'opérations du calculateur luimême. La programmation de ce dernier est alors modifiée de manière à ne stocker que la série des valeurs successives de $V$ la température n'étant 
relevée qu'avant et après la traversée. Ces valeurs sont stockées dans la mémoire centrale, ce qui réduit la période d'échantillonnage jusqu'à $178 \mathrm{~ms}$ et permet d'atteindre des durées d'enfoncement de $5 \mathrm{~s}$ à $2 \mathrm{~min}$. environ. Avec l'équipage mobile le plus léger, cela situe la limite inférieure des viscosités mesurables à moins de $10^{3} \mathrm{Pl}$.

4. Test et performances du dispositif expérimental. L'appareillage décrit au paragraphe précédent nous a permis d'étudier la viscosité de différents liquides surfondus tels que l'éthyl-2 hexanol-1 [19] et le carbonate de propylène [20]. On a constaté, dans les limites de l'appareillage $(4 \leqslant x \leqslant 24)$, que la valeur de la viscosité est bien indépendante du facteur géométrique $K$ (Fig. 2). Nous présentons ici les résultats obtenus avec le phényle salicylate, liquide dont la viscosité a été étudiée avec précision entre 60 et $-30^{\circ} \mathrm{C}$ par Jäntsch [21] et de 0 à $-60^{\circ} \mathrm{C}$ par Laughlin et Uhlmann [7]. Les techniques utilisées par ces derniers auteurs étaient basées sur la déformation d'une barre cylindrique (pour $\eta \gtrsim 10^{7} \mathrm{Pl}$ ) et la chute de bille (pour $\eta \lesssim 10^{7} \mathrm{Pl}$ ), en tenant compte dans ce cas d'un facteur correctif à la loi de Stokes dû aux effets de bord [22]. Le recoupement entre les deux méthodes était satisfaisant et la précision des mesures, variable selon les valeurs de $\eta$, comprise entre 5 et $20 \%$. Les résultats que nous présentons permettent donc de tester l'appareillage et de comparer les techniques de pénétrométrie et de flexion à basse température. On sait d'autre part qu'à haute température $\left(500-700^{\circ} \mathrm{C}\right)$ les mesures de viscosité effectuées par flexion d'éprouvettes et par allongement de fibres (verres silico-sodo-calciques) se sont révélées être bien concordantes [23].

4.1 LINÉARITÉ DE $h^{2}(t)$. - Les mesures sur le phényle salicylate ont été effectuées en gardant la même géométrie $\left(R_{1}=0,5 \mathrm{~mm}, x=24\right)$.

Un exemple typique des enregistrements obtenus lorsque la viscosité est inférieure à environ $10^{8} \mathrm{Pl}$ est donné figure 4. Le graphique $h^{2}(t)$ est linéaire pratiquement depuis l'origine $(h=0)$ jusqu'à quelques millimètres du fond où apparait alors un effet de bord qui n'est pas pris en compte dans le modèle théorique.

Pour des viscosités plus élevées, il apparaît une deuxième région non linéaire, située, elle, au début de l'enfoncement, et dont la présence peut être attribuée à un régime transitoire dépendant des propriétés viscoélastiques du liquide [7]. Au voisinage de $10^{10} \mathrm{Pl}$ le régime transitoire affecte $1 / 5$ de la traversée et sa durée n'excède pas $4 \%$ de la durée totale d'enfoncement. Dans tous les cas l'enregistrement $h^{2}(t)$ permet de délimiter graphiquement la zone où $h^{2}$ varie linéairement au cours du temps, et d'éliminer les régions affectées par le régime transitoire et les effets de bord. Les valeurs sélectionnées sont alors traitées suivant une régression linéaire pour en extraire la

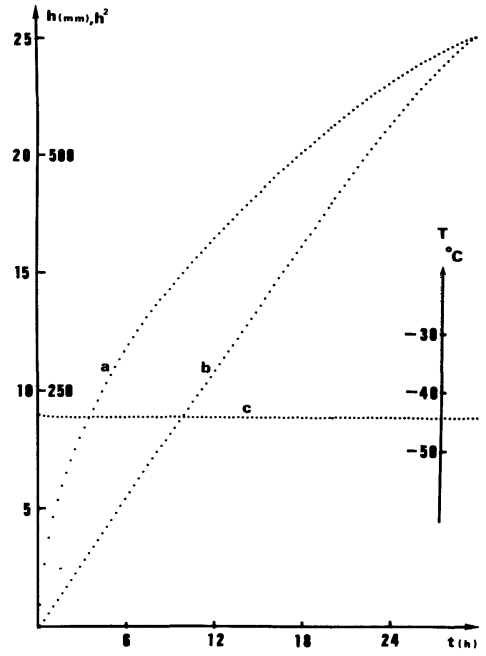

Fig. 4. - Exemple d'enregistrement obtenu au cours d'une mesure de viscosité par pénétrométrie : phényle salicylate à $-44,6^{\circ} \mathrm{C} ; \eta=3,8 \times 10^{7} \mathrm{Pl}$. a) $\left.\left.h(t) ; b\right) h^{2}(t) ; c\right) T(t)$. Coefficient de corrélation de $h^{2}(t): 0,9998$ pour $h<23 \mathrm{~mm}$.

[An example of recording used to measure the viscosity by penetrometer method : phenyl salicylate at $-44.6^{\circ} \mathrm{C}$, $\eta=3.8 \times 10^{7} \mathrm{Pl}$. a) $\left.\left.h(t) ; b\right) h^{2}(t) ; c\right) T(t)$. Correlation coefficient of $h^{2}(t): 0.9998$ when $h<23 \mathrm{~mm}$.]

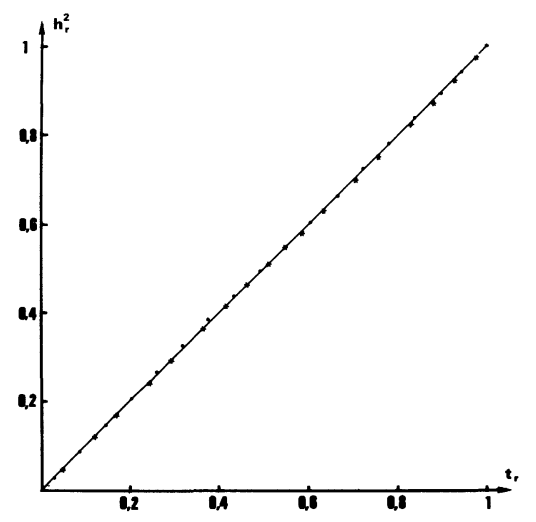

Fig. 5. - Courbes réduites $h_{\mathrm{r}}^{2}\left(t_{\mathrm{r}}\right)$ pour deux valeurs très différentes de la viscosité (phényle salicylate) : $\bullet \eta_{1}=$ $3,8 \times 10^{7} \mathrm{Pl}$, coefficient de corrélation $0,99991, * \eta_{2}=$ $3,8 \times 10^{3} \mathrm{Pl}$, coefficient de corrélation 0,999 93 .

[Reduced curves $h_{\mathrm{r}}^{2}\left(t_{\mathrm{r}}\right)$ for two very different values of viscosity (phenyl salicylate) : $\bullet \eta_{1}=3.8 \times 10^{7} \mathrm{Pl}$, correlation coefficient $0.99991, * \eta_{2}=3.8 \times 10^{3} \mathrm{Pl}$, correlation coefficient 0.999 93.

pente $b$. Le coefficient de corrélation est supérieur à 0,9991 . Ce résultat est illustré figure 5 où l'on a représenté en valeurs réduites $h_{\mathrm{r}}=h / \Delta h, t_{\mathrm{r}}=t / \Delta t$ ( $\Delta h$ et $\Delta t$ étendue et durée de la zone linéaire) les variations $h_{\mathrm{r}}^{2}\left(t_{\mathrm{r}}\right)$.

L'excellente linéarité de $h^{2}(t)$ confirme la validité du modèle théorique, le caractère newtonien du liquide, et constitue un test de la bonne stabilité thermique de l'appareillage. 
Tableau I. - Viscosité du phényle salicylate à basse température.

[Viscosity of phenyl salicylate at low temperature.]

\begin{tabular}{|l|c|c|c|c|c|c|c|}
\hline$T(\mathrm{~K})$ & 243,5 & 239,9 & 237,5 & 233,0 & 230,6 & 228,6 & 218,7 \\
\hline $\begin{array}{c}1000 / T \\
\left(\mathrm{~K}^{-1}\right)\end{array}$ & 4,11 & 4,17 & 4,21 & 4,29 & 4,34 & 4,38 & 4,57 \\
\hline$\eta(\mathrm{Pl})$ & $4,90 \times 10^{3}$ & $2,19 \times 10^{4}$ & $1,23 \times 10^{5}$ & $1,38 \times 10^{6}$ & $7,08 \times 10^{6}$ & $3,80 \times 10^{7}$ & $2,00 \times 10^{10}$ \\
\hline $\log \eta$ & 3,69 & 4,34 & 5,09 & 6,14 & 6,85 & 7,58 & 10,3 \\
\hline
\end{tabular}

4.2 Viscosité DU PHÉNYLE SAliCYLATE. - Les résultats obtenus entre 200 et $250 \mathrm{~K}$ sont donnés dans le tableau I.

Compte tenu des sondes utilisées, la lecture de $T$ peut être entachée d'une erreur maximale de $0,3 \mathrm{~K}$. Comme d'autre part il est difficile d'évaluer le facteur géométrique $K$ à mieux que $4 \%$, on peut estimer la précision relative sur la mesure de $\eta$ à environ 5 à $10 \%$ selon la zone de température.

Les variations de la viscosité en fonction de $T$ (Fig. 6), sont très proches du type d'Arrhénius, comme

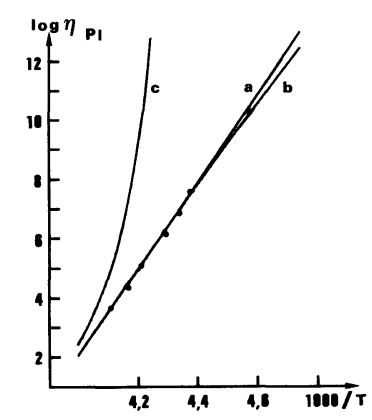

Fig. 6. - Variation thermique de la viscosité du phényle salicylate : • résultats obtenus par la méthode de pénétrométrie. a) Courbe $\log \eta=a+\frac{b}{T}+\frac{c}{T^{2}}$, les constantes étant obtenues par une méthode de moindres carrés à partir de nos résultats. b) Même relation avec les constantes tirées des résultats de Laughlin et Uhlmann [7]. c) Courbe $\log \eta=A+\frac{B}{T-T_{0}}, A, B$ et $T_{0}$ étant obtenues à partir des résultats de Laughlin et Uhlmann [7] et Jäntsch [21] à $T \geqslant 254 \mathrm{~K} A=-3,70, B=146, T_{0}=226,4 \mathrm{~K}$.

[Viscosity temperature dependence for phenyl salicylate : - data of present investigation by penetrometer method. a) Curve $\log \eta=a+\frac{b}{T}+\frac{c}{T^{2}}$, the constants being calculated by a least squares method from our data. $b$ ) The same relation with constants calculated from Laughlin and Uhlmann data [7]. c) Curve $\log \eta=A+\frac{B}{T-T_{0}}$. The values of $A, B, T_{0}$ are obtained with Laughlin and Uhlmann [7] and Jäntsch [21] data at $T \geqslant 254 \mathrm{~K}$ $A=-3.70, B=146, T_{0}=226.4 \mathrm{~K}$. $]$ l'avaient d'ailleurs signalé Laughlin et Uhlmann [7] On obtient cependant un meilleur ajustement (écarttype 0,092) en introduisant un paramètre supplémentaire, et en utilisant :

$$
\log \eta=a+b\left(\frac{10^{3}}{T}\right)+c\left(\frac{10^{3}}{T}\right)^{2} .
$$

Les coefficients déterminés par une méthode des moindres carrés ont les valeurs suivantes :

$$
a_{1}=-40,30, b_{1}=7,29, \quad c_{1}=0,826 .
$$

Pour comparer nos résultats à ceux de Laughlin nous avons ajusté leurs données suivant la même relation (10) et obtenu les valeurs :

$$
a_{2}=-69,24, \quad b_{2}=21,20, \quad c_{2}=-0,844 \text {. }
$$

Les courbes représentatives (Fig. $6 a$ et $b$ ) sont pratiquement confondues entre $10^{3}$ et $10^{8} \mathrm{Pl}$ (Tableau II). Au-delà, bien que les valeurs de la viscosité diffèrent (leur rapport peut atteindre 2,5 aux environs de $10^{12} \mathrm{Pl}$ ), la concordance entre (11) et (12) est encore très acceptable puisque les deux relations conduisent à des valeurs de la température de transition vitreuse 213,4 et $211,9 \mathrm{~K}$ très proches l'une de l'autre.

L'ensemble de ces résultats confirme aussi que l'extrapolation du régime de Vogel Fulcher [24, 25]

Tableau II. - Températures correspondant à diverses viscosités du phényle salicylate, calculées $\left(T_{1}\right)$ à partir de nos résultats et $\left(T_{2}\right)$ à partir de ceux de Laughlin et Uhlmann.

[Temperatures corresponding to some viscosities of phenyl salicylate, calculated $\left(T_{1}\right)$ from our data and $\left(T_{2}\right)$ from Laughlin and Uhlmann data.]

\begin{tabular}{|l|c|c|c|c|c|}
\hline \multicolumn{1}{|c|}{$\log \eta$} & 4 & 6 & \multicolumn{1}{c|}{8} & 10 & 12 \\
\hline$T_{1}(\mathrm{~K})$ & 241,70 & 233,77 & 226,45 & 219,68 & 213,40 \\
\hline$T_{2}(\mathrm{~K})$ & 241,80 & 233,78 & 226,15 & 218,88 & 211,94 \\
\hline$T_{1}-T_{2}(\mathrm{~K})$ & $-0,10$ & $-0,01$ & 0,30 & 0,80 & 1,46 \\
\hline
\end{tabular}


observé avec une très bonne précision dans la gamme $\eta \lesssim 10^{2} \mathrm{Pl}$ [7], n'est absolument pas valable (Fig. $6 c$ ) dans le domaine des viscosités élevées, supérieures à $10^{3} \mathrm{Pl}$.

5. Conclusion. - L'étude du phényle salicylate montre une bonne concordance entre les mesures de viscosité obtenues dans la gamme $10^{4}-10^{11} \mathrm{Pl}$ par flexion d'une éprouvette [7] et par pénétrométrie. Cette dernière technique semble d'application plus générale, car elle évite la réalisation d'éprouvettes de géométrie déterminée à basse température.

L'automatisation des mesures de pénétrométrie améliore incontestablement la précision, et leur traitement numérique permet d'analyser les variations de $h^{2}(t)$ et de localiser les zones non linéaires engendrées par le transitoire, les effets de bord, une dérive accidentelle de la température, ou une perturbation quelconque.

On peut noter que sous charge constante, la contrainte de cisaillement et la vitesse d'enfoncement diminuent au cours du temps et cela peut conduire à des durées d'enregistrement élevées. En contrepartie la linéarité de $h^{2}(t)$ observée sous contrainte variable, et l'invariance de $\eta$ par rapport à $F$ et $K$ constituent un test du caractère newtonien du liquide.

\section{Bibliographie}

[1] Harrison, G., The Dynamic Properties of Supercooled Liquids (Academic Press, London) 1976.

[2] ZARZYCKI, J., Les Verres et l'Etat Vitreux (Masson, Paris) 1982.

[3] Greet, R., Turnbull, D., J. Chem. Phys. 46 (1967) 1243.

[4] Ling, A., Willard, J., J. Phys. Chem. 72 (1968) 1918.

[5] Ling, A., Willard, J., J. Phys. Chem. 72 (1968) 3349.

[6] Tweer, H., Simmons, J., Macedo, P., J. Chem. Phys. 54 (1970) 1952.

[7] Laughlin, W., Uhlmann, D., J. Phys. Chem. 76 (1972) 2317.

[8] Wilson, W., Mellon, P., Berberian, J., J. Chem. Phys. 76 (1982) 2602.

[9] Wong, J., Angell, C., Glass Structure by Spectroscopy (M. Dekker, New York) 1976.

[10] Berberian, J., Rev. Sci. Instrum. 51 (1980) 1136.

[11] Van Wazer, J., Lyons, J., Kim, K., Colwell, R., Viscosity and Flow Measurement (Interscience, New York) 1963.

[12] Persoz, B., Vourlat, P., Viscosité, Techniques de l'Ingénieur 1967.
[13] Smith, T., Ferry, J., Schremp, F., J. Appl. Phys. 20 (1949) 144.

[14] Lawaczeck, F., Z. Ver, Deut. Ing. 63 (1919) 677.

[15] Bikerman, J., J. Colloid. Sci. 3 (1948) 75.

[16] Brun, E., Martinot-Lagarde, A., Mécanique des fluides, T. II (Dunod, Paris) 1968.

[17] LANDAU, L., LifChitz, E., Mécanique des fluides (Mir, Moscou) 1971.

[18] Catier, E., Electron. Indust. 11 (1981) 80.

[19] Jorat, L., Noyel, G., Bondeau, A., HuCK, J., Communication présentée au Congrès de la Société Française de Physique (Clermont-Ferrand) 1981. Colloque Verres et amorphes.

[20] Huck, J., Noyel, G., Jorat, L., Bondeau, A., J. Electrostatics 12 (1982) 221.

[21] JäNTSCH, O., Z. Kristallogr. 108 (1956) 185.

[22] Faxen, H., Ann. Phys. 68 (1922) 89.

[23] Hagy, H., J. Am. Ceram. Soc. 46 (1963) 93.

[24] Vogel, H., Physik Z. 22 (1921) 645.

[25] Fulcher, G., J. Am. Ceram. Soc. 8 (1925) 339. 